Published in final edited form as:

Am J Gastroenterol. 2017 May ; 112(5): 812-813. doi:10.1038/ajg.2017.67.

\title{
Reply to Song et al
}

\author{
Rena Yadlapati ${ }^{1}$, John E. Pandolfino ${ }^{1}$, and Bruce K. Tan ${ }^{2}$ \\ ${ }^{1}$ Division of Gastroenterology and Hepatology, Feinberg School of Medicine, Northwestern \\ University, Chicago, Illinois, USA \\ ${ }^{2}$ Division of Otolaryngology, Feinberg School of Medicine, Northwestern University, Chicago, \\ Illinois, USA
}

\section{To the Editor}

We thank you for the opportunity to respond to the comments of Song and colleagues ${ }^{12} \mathrm{We}$ agree that although the reflux symptom index (RSI) questionnaire is commonly used in clinical research to examine symptom severity, the RSI has several shortcomings as an outcome metric. The RSI is a cumulative score based on throat symptoms and heartburn. ${ }^{3}$ Throat symptoms include dysphonia, throat clearing, postnasal drip, globus, cough, dysphagia, and difficulty breathing. As mentioned by Song et al., the mechanisms and response to treatment for these individual laryngeal symptoms are diverse, and should be studied more precisely rather than lumped together in the RSI. Similarly, the editorial by Vaezi explains that the RSI is problematic when examining response to acid suppression since the RSI may improve based on an isolated response to heartburn without improvement in throat symptoms. ${ }^{4}$ The limitations with the RSI allude to the overarching problem with the nebulous and overused term larynopharyngeal reflux (LPR). While in some cases gastroesophageal reflux can cause laryngeal injury, nowadays, laryngeal complaints are often mistakenly equated with LPR, regardless of whether a true possibility of gastroesophageal reflux exists. Moving forward, we need to identify better outcome measures, separate out throat symptoms, redefine rigorous criteria for a diagnosis of LPR, and remind practitioners that all laryngeal complaints are not related to gastroesophageal reflux.

In our study, oropharyngeal $\mathrm{pH}$-monitoring results did not predict symptom response. Song and colleagues highlight that oropharyngeal $\mathrm{pH}$-metry does not measure weakly acidic or non-acidic reflux burden. Advancements in the field of gastroesophageal reflux disease now provide diagnostic tools to examine non-acid related properties of gastroesophageal reflux, and future research in extraesophageal syndromes should examine the role of these tests. For instance, salivary pepsin is a potentially sensitive and specific biomarker of

Corresponding Author: Rena Yadlapati, MD, Division of Gastroenterology \& Hepatology, Northwestern University Feinberg School of Medicine, 676 North St. Clair St. Suite 1400 Chicago, IL 60611, Office: (312) 926-7648; Fax: (312) 695-3999, rena.yadlapati@northwestern.edu.

Author Contributions: All authors (RY, JEP, BKT) contributed to drafting and finalization of manuscript.

Potential Conflict of Interest (Financial, Professional or Personal): None

Disclosures: RY: Supported by NIH T32DK101363 
gastroesophageal reflux ${ }^{5}$, and our prior research suggests that once appropriate thresholds are identified, salivary pepsin may be a reliable biomarker of reflux associated laryngeal symptoms. ${ }^{6}$ Mucosal impedance, a novel tool to measure direct conductivity of the epithelium, seems to have high specificity in distinguishing and predicting response to treatment in esophageal disorders and exploring the mucosal impedance of the laryngeal epithelium is intriguing. ${ }^{7}$

Finally, Song and colleagues mention the potential importance of post-PPI therapy oropharyngeal monitoring to examine physiologic changes between responder and nonresponder groups. Although our study was originally designed to study post-treatment oropharyngeal monitoring, only 7 of 34 subjects agreed to a second post-treatment oropharyngeal $\mathrm{pH}$ monitoring (5 non-responders, 2 complete responders, and 0 partial responders). The results by subject in table 1 depicts the low acid exposure across both groups with the exception of one complete responder and one non-responder.

We thank Song and colleagues for raising these important points. Although optimizing a clinical approach to suspected LPR is a major undertaking, we agree that next steps require clarification of diagnostic criteria and outcomes measures, and examination of non-acid reflux mechanisms.

\section{Abbreviations \\ RSI Reflux Symptom Index \\ LPR Laryngopharyngeal reflux}

\section{References}

1. Yadlapati R, Pandolfino JE, Lidder AK, et al. Oropharyngeal pH Testing Does Not Predict Response to Proton Pump Inhibitor Therapy in Patients with Laryngeal Symptoms. Am J Gastroenterol. 2016; 111:1517-24. [PubMed: 27091320]

2. Song L, Liao W, Lu Y, et al. Laryngeal reflux and symptoms need further elucidation using new techniques. American Journal of Gastroenterology. 2017

3. Belafsky PC, Postma GN, Koufman JA. Validity and reliability of the reflux symptom index (RSI). J Voice. 2002; 16:274-7. [PubMed: 12150380]

4. Vaezi MF. Editorial: Reflux and Laryngeal Symptoms: A Sea of Confusion. Am J Gastroenterol. 2016; 111:1525-7. [PubMed: 27808149]

5. Hayat JO, Gabieta-Somnez S, Yazaki E, et al. Pepsin in saliva for the diagnosis of gastrooesophageal reflux disease. Gut. 2015; 64:373-80. [PubMed: 24812000]

6. Yadlapati R, Adkins C, Jaiyeola DM, et al. Abilities of Oropharyngeal pH Tests and Salivary Pepsin Analysis to Discriminate Between Asymptomatic Volunteers and Subjects With Symptoms of Laryngeal Irritation. Clin Gastroenterol Hepatol. 2016; 14:535-42. e2. [PubMed: 26689899]

7. Vaezi MF, Choksi Y. Mucosal Impedance: A New Way To Diagnose Reflux Disease and How It Could Change Your Practice. Am J Gastroenterol. 2017; 112:4-7. [PubMed: 27958288] 
